\title{
LUT
}

Lappeenranta

University of Technology

\section{Optical properties of Bacteriorhodopsin-Gold Bionano Interfaces}

Das Subhabrata, Asmara Teguh Citra, Patra Abhirup, Song Zhaoning, Bista Sandip Singh, Somasundaran Ponisseril, Rusydi Andrivo, Barbiellini Bernardo, Venkatesan Renugopalakrishnan

This is a Post-print version of a publication

published by American Chemical Society

in The Journal of Physical Chemistry C

DOI: $10.1021 /$ acs.jpcc.9b06488

Copyright of the original publication: () 2019 American Chemical Society

Please cite the publication as follows:

Das S., Asmara T. C., Patra A., Song Z., Bista S. S., Somasundaran P., Rusydi A., Barbiellini B., Venkatesan R. (2019). Optical Properties of Bacteriorhodopsin-Gold Bionano Interfaces. The Journal of Physical Chemistry C. DOI: 10.1021/acs.jpcc.9b06488 


\section{Optical properties of Bacteriorhodopsin-Gold Bionano Interfaces}

Subhabrata Das", † Teguh Citra Asmara",, đ Abhirup Patra, ${ }^{\S}$ Zhaoning Song,"

Sandip Singh Bista, $\|$ Ponisseril Somasundaran, ${ }^{\dagger}$ Andrivo Rusydi, ${ }^{*, \perp}$ Bernardo Barbiellini, *,\#,@ and Renugopalakrishnan Venkatesan ${ }^{*, \triangle, \nabla}$

$\dagger$ Langmuir Center of Colloids and Interfaces, Columbia University in the City of New York, New York, NY 10027, USA

$\ddagger$ Department of Physics, National University of Singapore, 21 Lower Kent Ridge Rd, Singapore 119077

9Photon Science Division, Paul Scherrer Institute, CH-5232 Villigen PSI, Switzerland $\S$ School of Materials Science and Engineering, Georgia Institute of Technology, Atlanta,

$$
\text { GA } 30332
$$

||Department of Physics and Astronomy,University of Toledo, $\mathrm{OH}$ 43606, United States

$\perp$ Department of Physics, National University of Singapore

\#Department of Physics, LUT University, FI-53851 Lappeenranta, Finland

@ Department of Physics,Northeastern University, Boston, Massachusetts 02115, USA

$\triangle$ Department of Chemical Biology, Center for Renewable Energy, Northeastern University, Boston, Massachusetts 02115, United States

$\nabla$ Boston Children's Hospital, Harvard Medical School, Boston, MA 02115, USA

E-mail: phyandri@nus.edu.sg; bernardo.barbiellini@lut.fi; v.renugopalakrishnan@northeastern.edu

"Equal Contribution

Abstract 
Monolayers of different mutants of Bacteriorhodopsin(bR) with purple membranes are deposited on gold $(\mathrm{Au})$ substrates. Optical measurements and density functional theory based electronic structure calculations are reported for the first time for the bR chromophore, which is the retinal protonated Schiff base. The dielectric function of the chromophore is extracted using Spectroscopic Ellipsometry. Designed mutants of $\mathrm{bR}$ are used to investigate the bonding with the gold substrate and bR-Au bonding changes can be tracked using the optical properties of the retinal fragment.

\section{Introduction}

Intriguing problems are often unravelled at the interfaces of disparate materials as promulgated by Herbert Kroemer by stating that the interface is the device. ${ }^{1}$ Beyond semiconductors, the phenomenon is also of relevance to proteins adsorbed on solid surfaces in biomaterials field as well as nanotechnology field. Protein-surface interactions are central to exploiting the interfacial protein constructs, such as transducers, sensors and other biological/electronic junction based functional components. ${ }^{2,3}$ While $1 \mathrm{D}$ structures on the centimeter scale could be readily fabricated, the scaling up of this approach to higher dimensions and to shorter wavelengths could enable the controllable assembly of complex phononic band gap materials on the hundreds of microscale. ${ }^{4}$ Nanoparticle/biological interfaces facilitate the molecular adsorption driven protein corona formation and biocatalytic processes under the influence of colloidal and dynamic biophysicochemical interactions. ${ }^{5}$ Molecular mechanisms, including extensive dynamics that have been less explored often mediate such protein interfaces. Prior investigation have often focused on "hot spots" which are an exclusive subset of all interface residues instead of the whole protein molecules. ${ }^{6}$ These interaction hot spots are pivotal to protein-protein recognition and binding, and retard such interactions on mutation. These engineered interaction hot-spots on active surfaces are difficult to capture to

enable their augmentation into optoelectronic devices. ${ }^{7-9}$ For instance, Bio-sensitized Solar Cells (BSSCs) as a possible alternative to Silicon based cells and Dye-sensitized solar cells 
(DSSCs), are promising development towards providing environmentally-friendly and stable devices. The requirements for the bio-sensitizer in solar cell application are thermal robustness over the operating temperature regime, interfacial compatibility to facilitate charge transport while absorbing the solar radiation, and stability under environmental conditions. Antenna proteins like Xanthorhodopsin are typically associated with the initial stages of photosynthesis and play the role of collecting solar radiation. Thus, they are of interest for BSSC applications. ${ }^{10,11}$

We recall that Bacteriorhodopsin (bR), a 7 helical protein, found in the archaea, Halobacterium salinarium, functioning as a natural light driven proton pump, ${ }^{12,13}$ is another promising environmentally benign alternative to toxic dyes for usage in BSSCs. bR is a thermophilic protein manifesting stability against high salt concentration (up to $5 \mathrm{M} \mathrm{NaCl}$ ) in aqueous state and up to $140{ }^{\circ} \mathrm{C}$ in dried state yielding high quantum photon to electron conversion efficiency (QE) of $66 \%$ for all the visible spectrum. Upon photoactivation, the transport of electrons toward the Schiff base from the $\beta$ ionone ring of the bR chromophore is initiated. This electronic shift is comparable to the displacement of a single electron at $2.6 \AA$ down the polyene chain in $\mathrm{bR}$ chromophore in a reverse direction to the initial photo-voltage spike accompanied by a large change in dipole moment. ${ }^{14}$ This charge shift happens transiently and the photoexcitation induced voltage has so far eluded experimental detection. However, this electron density displacement is critical as it propels all subsequent events resulting in the bR chromophore photoisomerization. The charge separation and electron ejection are the primary events which are critical for the application of bR as an efficient light energy harvesting system.

The retinal inside bR triggers a photocycle upon absorption of light at $\sim 570 \mathrm{~nm}$, leading to proton pumping to the external medium across the transmembrane from the cytoplasm inside the cells. The function of bR for light absorption is based upon a sequence of photochemical reactions. The protein-chromophore complex undergoes photon-activated conformational changes from the all-trans to the 13-cis. High-speed atomic force microscopy has 
been used to capture such conformational changes in photo-activated D96N bR mutant ${ }^{15}$ which has a longer photocycle compared to the Wild-type (WT) bR. Structural flexibility studies of bR at micro to millisecond timescales with techniques based on AFM have given further insights onto force-induced deformations across the bR structure. ${ }^{16}$ The chromophore evolution induces rapid transition from the K590 to the L550 state. A proton is released in the slow transition from the L550 state to the M410 state. bR electronic junctions display different conduction properties in the dark and in the presence of light ${ }^{17}$ as explained by models using the electron tunnel effect. ${ }^{18}$ There have been numerous attempts to design devices utilizing the physical properties of bR. ${ }^{19}$ Nonlinear photonic bandgap properties have already been attributed to $\mathrm{bR} .^{20}$ In particular, an efficient and stable photocurrent response from a bR film using an electrochemical sandwich-type photovoltaic junction structure of $\mathrm{TiO}_{2} / \mathrm{bR} /$ electrolyte/Al electrode has been recently obtained. ${ }^{2}$ To understand the photovoltaic characteristics, the knowledge of electronic structure of photoactive material within the device is crucial. There are two mechanisms to explain the optical excitation to photocurrent conversion; direct transport of electrons through bR polyene chain ${ }^{18}$ leading to photo-current enhancement; and Au layer de-excitation by virtue of Auger mediated Sticking (AMS). ${ }^{21}$ This leads to an efficient ballistic electron production and subsequent transport in the $\mathrm{Au}$ layer and captured by the semiconductor layer underneath. It is expected that the latter process will dominate in the operation of the BSSCs. The interaction of gold with bR, especially the chemical bonds between $\mathrm{Au}$ and $\mathrm{bR}$, remains poorly understood due to the inert nature of bulk gold. Hence, characterization of the optical property of $\mathrm{bR} / \mathrm{Au}$ interface is the principal motivation of this paper.

A direct method for probing the electronic band structure of thin film materials is to measure their complex dielectric response using spectroscopic ellipsometry. ${ }^{22}$ Here, we use this technique to isolate the optical spectrum of the retinal fragment of bR deposited on Au surface by measuring its complex dielectric function within photon energies of $0.5-5.9 \mathrm{eV} .{ }^{23}$ We find the edge of an optical transition of the WT bR and Cys bR mutant at around 2.30 
$\mathrm{eV}(539 \mathrm{~nm})$, with a peak at around $2.55 \mathrm{eV}(486 \mathrm{~nm})$, making bR suitable to capture solar irradiation in the visible range. The ellipsometry results are supported by ab-initio density functional theory (DFT) calculations.

\section{Materials and Methods}

\section{Attachment of bR to Au substrate}

WT bR and specially designed Cys36 bR,D96N bR mutants were immobilized on Au substrate by covalent attachment of Cys Sulphydryl groups to the Au substrate. A $6.67 \mathrm{~g} / \mathrm{L}$ bR solution was diluted 10 folds in suspension buffer $(150 \mathrm{mM} \mathrm{KCl}, 10 \mathrm{mM}$ Tris-HCl, $\mathrm{pH}$ 8.2) to a final absorbance of $0.2-0.3$ at wavelength of $570 \mathrm{~nm} .0 .5 \mathrm{~mL}$ of this diluted bR solution in suspension buffer was blended with $250 \mu \mathrm{L}$ of incubation buffer $(300 \mathrm{mM} \mathrm{KCl}$, $10 \mathrm{mM}$ Tris-HCl, $\mathrm{pH}$ 7.82) to prepare samples for characterization. A $60 \mathrm{~nm}$ Au layer was thermally evaporated onto glass substrates. Then, the bR films were deposited on Au coated glass substrates uniformly by spin coating the bR solution in a suspension buffer solution with a concentration of $0.1 \mathrm{wt} \%$ at $1000 \mathrm{rpm}$ for $30 \mathrm{~s}$. The technique exploits the ability of a sulfhydryl group of the Cys mutant to covalently bind to a gold surface, or to a heterobifunctional cross-linker bound to a surface. In this way, we obtained well-oriented bR monolayers containing purple membrane patches deposited on Au substrates (See Fig. S1 in Supplementary Information). Thus, we significantly improve the sample suitability for Ellipsometry measurements compared to samples described in a previous report. ${ }^{21}$

\section{Spectroscopic Ellipsometry}

Spectroscopic ellipsometry measurements are performed on WT, Cys and D96N bR layers each deposited on Au substrate (Fig. 1). The experiments are performed with a specular reflection geometry from 0.6 to $5.9 \mathrm{eV}$ using Woollam V-VASE ellipsometer at $50^{\circ}, 60^{\circ}$ and $70^{0}$ incident angles from the surface normal of the $\mathrm{bR} / \mathrm{Au}$ samples at room temperature, 


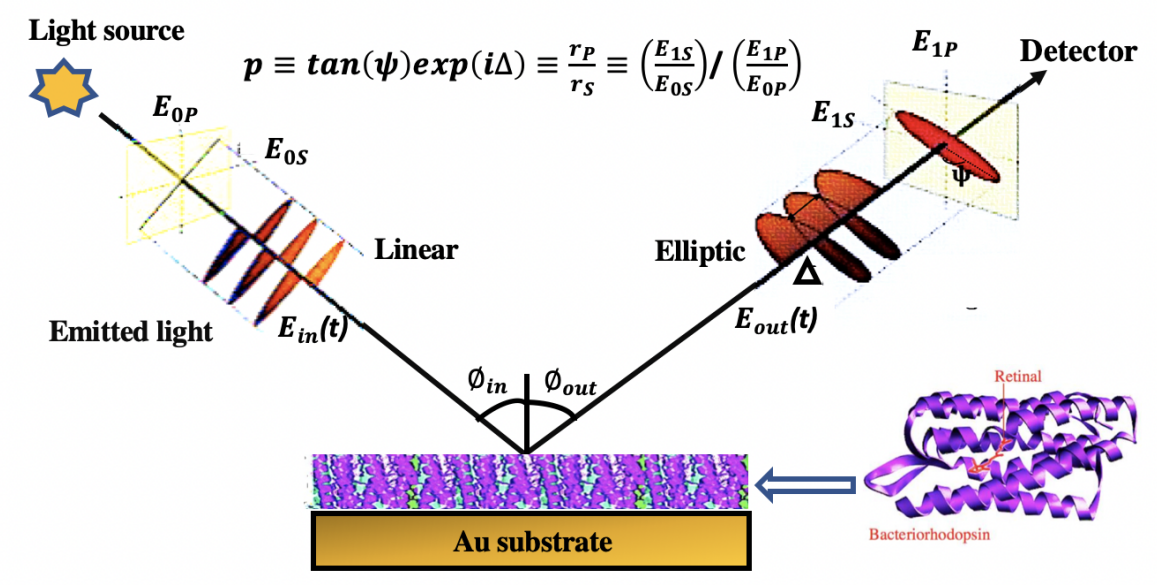

Figure 1: Schematic of the in situ spectroscopic ellipsometry setup with broadband illumination from top.

similar to the method described in literature. ${ }^{22}$ The beam spot size is focused down to approximately $0.5-1 \mathrm{~mm}$ using focusing lenses to minimize the effects of surface roughness and thickness non-uniformity, if any. Alongside the bR/Au samples, the bare Au substrate is also measured as the reference sample. The resulting ellipsometric parameters of each sample are analysed to extract the complex dielectric function, $\varepsilon(\omega)=\varepsilon_{1}(\omega)+i \varepsilon_{2}(\omega)$, of the bR layer from the raw data using Woollam WVASE analysis software. Since the samples are bR thin films deposited on $\mathrm{Au}$, they are modeled as a two-layer system. The $\varepsilon(\omega)$ of both the bR layers and the Au substrate are fitted using a combination of Drude and Herzinger-Johs PSemi-Tri oscillators, ${ }^{24}$ both of which are Kramers-Kronig transformable. To account for the surface ( $8 \mathrm{~nm}$ for Cys, $6 \mathrm{~nm}$ for WT) and interface (4 nm for both) roughnesses, a Bruggeman-mode effective medium approximation is used. The fitting is performed until the mean-squared error (MSE) between fitted and experimental data is minimized (see Supplementary Fig. S3). The resulting spectra of the bR samples are shown in Fig. 2(a) and Fig. 2(b) while that of bare Au substrate is in Supplementary Fig. S2. The $\varepsilon(\omega)$ of the bare Au substrate is consistent with previous reports. ${ }^{25}$ The angle-dependent analysis ${ }^{26,27}$ also reveals that the thicknesses of the Cys and WT bR layer are around $40 \mathrm{~nm}$ and $45 \mathrm{~nm}$, respectively. 


\section{Density Functional Theory Calculations}

The analysis of atomic projected density of states (pDOS) and the frequency dependent dielectric constant of the pristine molecule and the conjugate system gives us the nature of electronic, dielectric, and optical properties. We performed density functional theory (DFT) calculations within the Kohn-Sham scheme ${ }^{28,29}$ implemented in the Vienna ab Initio Simulation Program (VASP). ${ }^{30-32}$ At first, we relax the free retinal part in a box of size

$20 \times 21 \times 22 \AA^{3}$ with periodic boundary conditions using Perdew-Burke-Ernzerhof $(\mathrm{PBE})^{33}$ GGA functional. The ion-electron interaction is modeled using the $\mathrm{PAW}^{34}$ pseudopotential. To model the Gold substrate, we used a $c(6 \times 6) \mathrm{Au}(110)$ surface slab with $15 \AA$ vacuum (see top view of the slab in 4(a)). We find vacuum length larger than $15 \AA$ have negligible effect on the molecular optical properties. Lattice constant of the $\mathrm{Au}$ is determined fitting the Birch-Murnaghan equations of state (BM-EOS) to the energy-volume data. ${ }^{35}$ The moleculemetal systems were optimized by relaxing the top three layers of the metallic slab and the molecule. A kinetic-energy cutoff of $450 \mathrm{eV}$ is used for the wave functions, and Brillouin zone is sampled using a $\Gamma$ centered $6 \times 6 \times 1$ k-points for the slabs and $1 \times 1 \times 1$ for the molecule. $\mathrm{Ab}$ initio methods have been used in literature ${ }^{36}$ to characterize the ground and first excited state of the chromophore in the rhodopsin family containing retinal protonated Schiff base with five double bonds capable of undergoing photoisomerization.

\section{Results and discussion}

\section{Measurement of Dielectric spectrum using Spectroscopic Ellipsome- $\operatorname{try}$}

Figs. 2(a) and 2(b) shows the real, $\varepsilon_{1}(\omega)$ and imaginary, $\varepsilon_{2}(\omega)$ parts of dielectric spectrum, $\varepsilon(\omega)$ for the Cys and WT bR. In particular, $\varepsilon_{2}(\omega)$, is equivalent to the absorption spectrum, from which we can determine the optical excitations of the bR samples including their bandgap. 
As seen, an edge for both Cys and WT bR samples is found around $2.30 \mathrm{eV}(539 \mathrm{~nm})$, with a first excitation feature that peaks around $2.55 \mathrm{eV}(486 \mathrm{~nm})$, making it ideal to capture solar irradiation in the brightest parts of the visible range for solar cell applications. The shape of this first excitation peak (sharp and asymmetric) is typical of that of an exciton, indicating that the $2.30 \mathrm{eV}$ feature is a direct optical transition and that the charge separation mechanism in solar cells based on these samples would be mainly governed by excitonic interactions similar to that of other organic-based solar cells. ${ }^{37}$

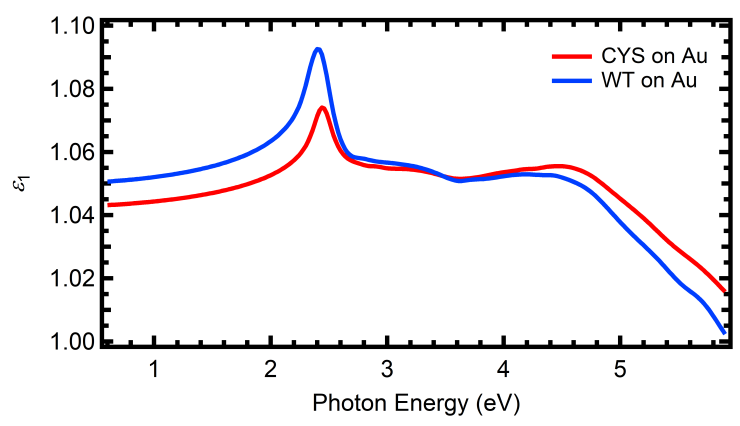

(a)

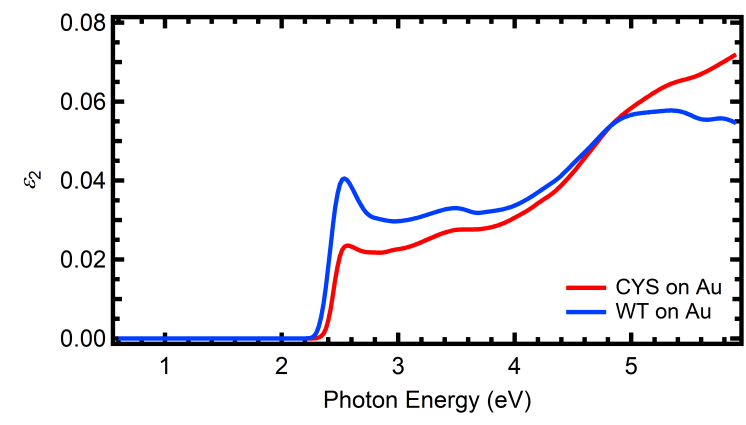

(b)

Figure 2: (a) The real part of complex dielectric function, $\varepsilon_{1}(\omega)$ of Cys-bR and WT-bR, (b) the imaginary part of complex dielectric function, $\varepsilon_{2}(\omega)$ of Cys-bR and WT-bR.

Interestingly, despite their distinct shapes and clear features, the absolute values of $\varepsilon_{1}(\omega)$ and $\varepsilon_{2}(\omega)$ of both bR samples is very close to 1 (only reaching up to 1.10) and 0 (only reaching up to 0.08), respectively, even above the bandgap, which means that their $\varepsilon(\omega)$ spectra are quite delicate and very similar to that of ambient air. For comparison, the $\varepsilon(\omega)$ values of typical inorganic insulators are at least one order of magnitude higher, like $\mathrm{SrTiO}_{3}$ whose absolute values of $\varepsilon_{1}(\omega)$ and $\varepsilon_{2}(\omega)$ are up to 10 and 8 , respectively, in the same energy range. ${ }^{22}$ These delicate $\varepsilon(\omega)$ values make the ellipsometry analysis of the bR samples very sensitive to the sample quality, because even a small amount of imperfections and depolarization effects can easily overshadow the effects of the actual $\varepsilon(\omega)$ on the raw ellipsometry data. For example, we also attempted to measure the D96N mutant of bR using ellipsometry, but the D96N sample has high depolarization effects $(\geq 4 \%)$ that massively 
overshadow the fitted $\varepsilon_{2}(\omega)$ (see Fig. S4). For comparison, the depolarizations of Cys and WT samples are very low at $\approx 1 \%$. Our ability to reliably extract the $\varepsilon(\omega)$ spectra of the Cys-bR and WT-bR samples is thus indicative of the high quality of these bR samples (as also indicated by their low depolarization), as well as the high sensitivity that ellipsometry has over other optics-based techniques. The most obvious trend in Figs. 2(a) and 2(b) is the weakening of the peaks when the bR-Au coupling increases as in the case of Cys-bR. This observation implies that the oscillator strength of the main optical transition decreases when the bR-Au coupling increases. In the next section, we will try to rationalize this behavior by using simulations based on density functional theory.

\section{Calculation of Dielectric spectrum using Density Functional Theory}

The optical properties of the retinal part of bR molecule are computed by using the mBJ meta-GGA ${ }^{38}$ exchange-correlation approximation within density functional perturbation theory (DFPT) as an extension of the preliminary calculations. ${ }^{39}$ To compute the atom projected density of states (DOS) of the free retinal part and the gold surface (as presented in Fig. 3, we performed PBE calculations on the relaxed geometry using a denser $k$-mesh. The retinal and the gold surface are illustrated in Fig. 4(a).

From Fig. 3(a), clear peaks of the molecular orbitals can be seen for the gas phase retinal molecule while the HOMO-LUMO gap of the pristine retinal disappears when the retinal is in close proximity with the Au substrate and a continuum forms upon strong hybridization with the substrate $d$ bands in conjugate system. This effect is a strong evidence that the delocalized $\pi$-electrons of the molecule plays an important role determining the ground state electronic properties. The dielectric function $\varepsilon_{2}(\omega)$ plot of the pristine retinal in Fig. 4(a) is in good agreement with the Spectroscopic Ellipsometry measurement for the WT bR but the agreement is poorer for the Cys mutant because of the Cys' stronger binding with the Au substrate. In detail, the computed peak of $\varepsilon_{2}(\omega)$ is 1.35 for the real part and 0.60 for the imaginary part respectively. The dielectric function plot of the free molecule itself agreeing 

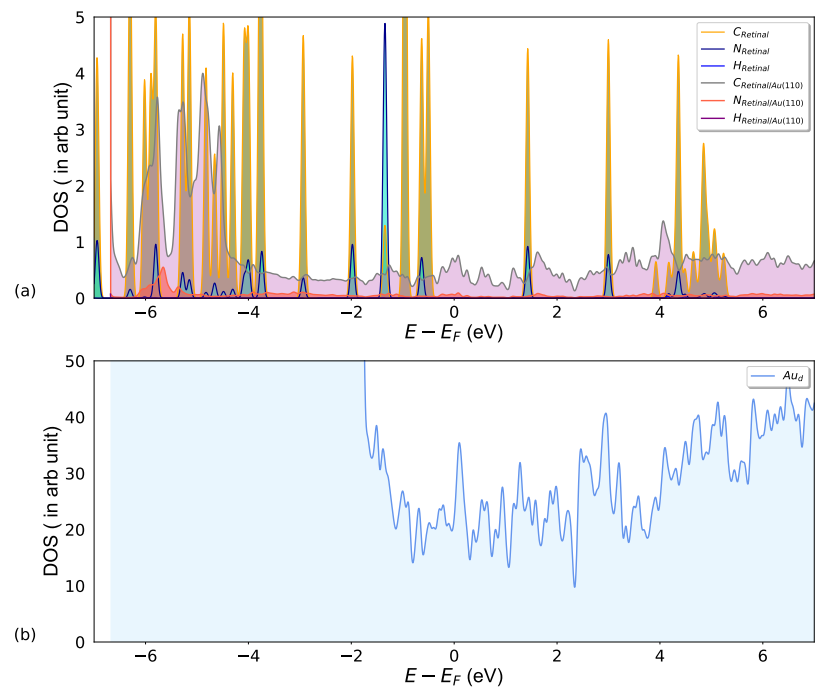

Figure 3: Atom-projected DOS computed using PBE functional with an effectively densed k-mesh for the relaxed geometries: (a) DOS of free retinal and the adsorbed retinal on gold surface, (b) PDOS of Au-d orbitals.

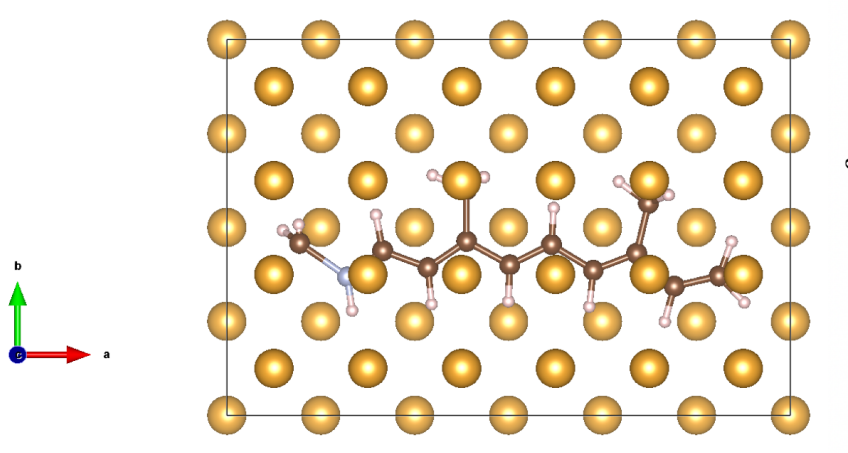

(a)

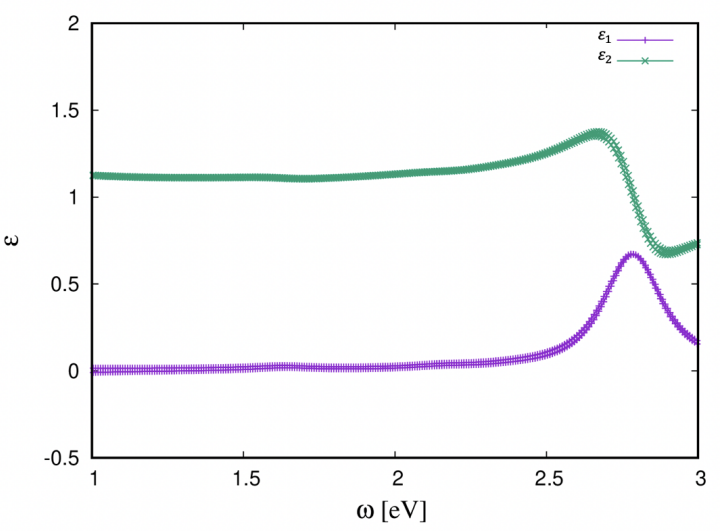

(b)

Figure 4: (a) Top view of the $\mathrm{Au}(110)$ surface slab with retinal part of $\mathrm{bR}$ used in the DFT calculations, (b) plot of Real $\left(\varepsilon_{1}\right)$ and Imaginary part $\left(\varepsilon_{2}\right)$ of the frequency dependent dielectric constant $\varepsilon(\omega)$ of free Retinal part calculated using the mBJ functional. 
with that of the less bound $\mathrm{bR}$ reveals the fact that coupling between the $\pi$ - electrons and the proton environment of the Schiff base plays the important role in modifying the dielectric spectrum. In fact, the charge transfer from metallic filled bands to LUMO of molecule delocalizes electron towards Schiff base and considerably alters the electronic structure of the adsorbed molecule.

\section{Conclusion}

We have studied the bR-Au Bionano interface relevant for advanced photonic and photovoltaic applications. We have shown that spectroscopic ellipsometry provides an elegant way to extract the optical properties of the retinal chromophore at the bR-Au interface. The bR$\mathrm{Au}$ bonding drives changes in the dielectric function of the retinal. Therefore, our study provides an original approach for exploring the bonding trends between gold and $\mathrm{bR}$ and opens up possibilities for an incisive bR-Au interface characterization using the oscillator strength of the main optical transition of the retinal chromophore to monitor the nature of the bR-Au coupling.

\section{Acknowledgements}

The authors thank the Singapore Synchrotron Light Source (SSLS) for providing the facility necessary for conducting the spectroscopic ellipsometry experiments. SSLS is a National Research Infrastructure under the Singapore National Research Foundation (NRF).SD acknowledges comments from Dr. Congcong Wu, Prof. Shashank Priya from Penn State University, Prof. Sakthi Kumar from Toyo University and Dr. Xingyu Gao from Chinese Academy of Sciences which helped improving the manuscript. T. C. Asmara and A. Rusydi are supported by the Singapore NRF under its Competitive Research Funding (No. NRF-CRP 8-2011-06 and No. R-398-000-087-281), MOE-AcRF Tier-2 (MOE2015-T2-1-099, MOE2015-T2-2-065, and MOE2015-T2-2-147), NUS YIA, and FRC (R-144-000-368-112, R- 
144-000-346-112, and R-144-000-364-112). AP acknowledges XSEDE start-up grant TGDMR190025 for the computational resources. C.W. and S.P. acknowledge the support from Air Force Office of Scientific Research (AFOSR) through grant number FA9550-18-1-0233 (Biophysics and Natural Materials). B.B. benefitted form support of the U.S. Department of Energy, Office of Science, Basic Energy Sciences contract no. DE-FG02-07ER46352, and wishes to acknowledge CSC -IT Center for Science, Finland, for computational resources. VR thanks Wallace Coulter Foundation, Rothschild Foundation for support.

\section{Supporting Information Available}

The procedure of synthesizing, cloning and expressing bR mutants; Surface Characterization of Bacteriorhodopsin-Gold surface using AFM and Confocal Microscopy; Spectroscopic Ellipsometry of pure Au substrate, and the Sensitivity of spectroscopic ellipsometry to sample quality.

\section{References}

(1) Kroemer, H. Quasi-electric fields and band offsets: teaching electrons new tricks. International Journal of Modern Physics B 2002, 16, 677-697.

(2) Thavasi, V.; Lazarova, T.; Filipek, S.; Kolinski, M.; Querol, E.; Kumar, A.; Ramakrishna, S.; Padrós, E.; Renugopalakrishnan, V. Study on the feasibility of bacteriorhodopsin as bio-photosensitizer in excitonic solar cell: a first report. Journal of nanoscience and nanotechnology 2009, 9, 1679-1687.

(3) Kaji, T.; Kasai, K.; Haruyama, Y.; Yamada, T.; Inoue, S.-I.; Tominari, Y.; Ueda, R.; Terui, T.; Tanaka, S.; Otomo, A. Enhanced photocurrent generation from bacteriorhodopsin photocells using grating-structured transparent conductive oxide electrodes. Journal of nanoscience and nanotechnology 2016, 16, 3206-3212. 
(4) Bishop, K. J. Acoustic metamaterials: Living bandgaps. Nature materials 2017, 16, 786.

(5) Nel, A. E.; Mädler, L.; Velegol, D.; Xia, T.; Hoek, E. M.; Somasundaran, P.; Klaessig, F.; Castranova, V.; Thompson, M. Understanding biophysicochemical interactions at the nano-bio interface. Nature materials 2009, 8, 543.

(6) Audette, G. F.; Lombardo, S.; Dudzik, J.; Arruda, T. M.; Kolinski, M.; Filipek, S.; Mukerjee, S.; Kannan, A. M.; Thavasi, V.; Ramakrishna, S. Protein hot spots at bionano interfaces. Materials Today 2011, 14, 360 - 365.

(7) Barbiellini, B.; Das, S.; Renugopalakrishnan, V.; Somasundaran, P. Electromagnetic Field in Hybrid Quantum Plasmonic-Photonic Systems. Condensed Matter 2018, 3, 10.

(8) Li, Y.-T.; Tian, Y.; Tian, H.; Tu, T.; Gou, G.-Y.; Wang, Q.; Qiao, Y.-C.; Yang, Y.; Ren, T.-L. A Review on Bacteriorhodopsin-Based Bioelectronic Devices. Sensors 2018, 18,1368 .

(9) Wagner, N. L.; Greco, J. A.; Ranaghan, M. J.; Birge, R. R. Directed evolution of bacteriorhodopsin for applications in bioelectronics. Journal of The Royal Society Interface 2013, 10, 20130197.

(10) Somasundaran, P.; Chin, M.; Latosiewicz, U. T.; Tuller, H. L.; Barbiellini, B.; Renugopalakrishnan, V. Nanoscience and Engineering for Robust Biosolar Cells. 2011.

(11) Das, S.; Wu, C.; Song, Z.; Hou, Y.; Koch, R.; Somasundaran, P.; Priya, S.; BarbielliniAmidei, B.; Venkatesan, R. Bacteriorhodopsin Enhances Efficiency of Perovskite Solar Cells. ACS applied materials \& interfaces $\mathbf{2 0 1 9}$

(12) Stoeckenius, W.; Lozier, R. H.; Bogomolni, R. A. Bacteriorhodopsin and the purple 
membrane of halobacteria. Biochimica et Biophysica Acta (BBA)-Reviews on Bioenergetics 1979, 505, 215-278.

(13) Haupts, U.; Tittor, J.; Oesterhelt, D. Closing in on bacteriorhodopsin: progress in understanding the molecule. Annual review of biophysics and biomolecular structure 1999, 28, 367-399.

(14) Wang, G.; Porschke, D. Dipole Reversal in Bacteriorhodopsin and Separation of Dipole Components. The Journal of Physical Chemistry B 2003, 107, 4632-4638.

(15) Shibata, M.; Yamashita, H.; Uchihashi, T.; Kandori, H.; Ando, T. High-speed atomic force microscopy shows dynamic molecular processes in photoactivated bacteriorhodopsin. Nat Nano 2010, 5, 208-212.

(16) Dong, M.; Husale, S.; Sahin, O. Determination of protein structural flexibility by microsecond force spectroscopy. Nature nanotechnology 2009, 4, 514.

(17) Li, L. S.; Xu, T.; Zhang, Y. J.; Jin, J.; Li, T. J.; Zou, B.; Wang, J.-P. Photovoltaic characteristics of $\mathrm{BR} / \mathrm{p}$-silicon heterostructures using surface photovoltage spectroscopy. Journal of Vacuum Science \& Technology A: Vacuum, Surfaces, and Films 2001, 19, $1037-1041$.

(18) Alfinito, E.; Reggiani, L. Charge transport in bacteriorhodopsin monolayers: The contribution of conformational change to current-voltage characteristics. EPL (Europhysics Letters) 2009, 85, 68002.

(19) Hampp, N. Bacteriorhodopsin as a Photochromic Retinal Protein for Optical Memories. Chemical Reviews 2000, 100, 1755-1776, PMID: 11777419.

(20) Clays, K.; Elshocht, S. V.; Persoons, A. Bacteriorhodopsin:a natural (nonlinear) photonic bandgap material. Opt. Lett. 2000, 25, 1391-1393. 
(21) Renugopalakrishnan, V.; Barbiellini, B.; King, C.; Molinari, M.; Mochalov, K.; Sukhanova, A.; Nabiev, I.; Fojan, P.; Tuller, H.; Chin, M. Engineering a Robust Photovoltaic Device with Quantum Dots and Bacteriorhodopsin. The Journal of Physical Chemistry C 2014, 118, 16710-16717, PMID: 25383133.

(22) Asmara, T.; Annadi, A.; Santoso, I.; Gogoi, P.; Kotlov, A.; Omer, H.; Motapothula, M.; Breese, M.; Rübhausen, M. Mechanisms of charge transfer and redistribution in LaAlO 3/SrTiO 3 revealed by high-energy optical conductivity. Nature communications $\mathbf{2 0 1 4}$, 5, 3663 .

(23) Das, S.; Asmara, T. C.; Song, Z.; Rusydi, A.; Barbiellini, B.; Somasundaran, P.; Renugopalakrishnan, V. Probing Biophysicochemical Interactions at Nano-Bio Interface of Perovskite Tandem Biosolar Cells. Biophysical Journal 2019, 116, 577a.

(24) Johs, B.; Herzinger, C.; Dinan, J.; Cornfeld, A.; Benson, J. Development of a parametric optical constant model for $\operatorname{Hg} 1-\mathrm{xCdxTe}$ for control of composition by spectroscopic ellipsometry during MBE growth. Thin Solid Films 1998, 313, 137-142.

(25) Olmon, R. L.; Slovick, B.; Johnson, T. W.; Shelton, D.; Oh, S.-H.; Boreman, G. D.; Raschke, M. B. Optical dielectric function of gold. Physical Review B 2012, 86, 235147.

(26) Asmara, T. C.; Santoso, I.; Rusydi, A. Self-consistent iteration procedure in analyzing reflectivity and spectroscopic ellipsometry data of multilayered materials and their interfaces. Review of Scientific Instruments 2014, 85, 3663.

(27) Fujiwara, H. Spectroscopic ellipsometry: principles and applications; John Wiley \& Sons, 2007.

(28) Hohenberg, P.; Kohn, W. Inhomogeneous Electron Gas. Phys Rev 1964, 136.

(29) Kohn, W.; Sham, L. J. Self-Consistent Equations Including Exchange and Correlation Effects. Phys. Rev. 1965, 140, A1133. 
(30) Kresse, G.; Hafner, J. Ab initio molecular dynamics for liquid metals. Phys. Rev. B $1993,47,558$.

(31) Kresse, G.; Hafner, J. Norm-conserving and ultrasoft pseudopotentials for first-row and transition elements. Phys. Rev. B 1994, 49, 14251.

(32) Kresse, G.; Joubert, D. From ultrasoft pseudopotentials to the projector augmentedwave method. Physical Review B 1999, 59, 1758.

(33) Perdew, J. P.; Burke, K.; Ernzerhof, M. Phys. Rev. Lett. 1996, 77, 3865.

(34) Blöchl, P. E. Projector augmented-wave method. Phys. Rev. B 1994, 50, 17953.

(35) Patra, A.; Bates, J. E.; Sun, J.; Perdew, J. P. Properties of real metallic surfaces: Effects of density functional semilocality and van der Waals nonlocality. Proc Nat Acad Sci 2017, 114, E9188.

(36) Ben-Nun, M.; Molnar, F.; Schulten, K.; Martínez, T. J. The role of intersection topography in bond selectivity of cis-trans photoisomerization. Proceedings of the National Academy of Sciences 2002, 99, 1769-1773.

(37) Gregg, B. A. Excitonic solar cells: The physics and chemistry of organic-based photovoltaics; ACS Publications, 2003.

(38) Tran, F.; Blaha, P. Accurate Band Gaps of Semiconductors and Insulators with a Semilocal Exchange-Correlation Potential. Phys. Rev. Lett. 2009, 102, 226401.

(39) Das, S.; Barbiellini, B.; Somasundaran, P.; Renugopalakrishnan, V. Density Functional Theory calculations of optical properties of Hybrid Halide Perovskite Bio-Solar Cells. APS Meeting Abstracts. 2019. 

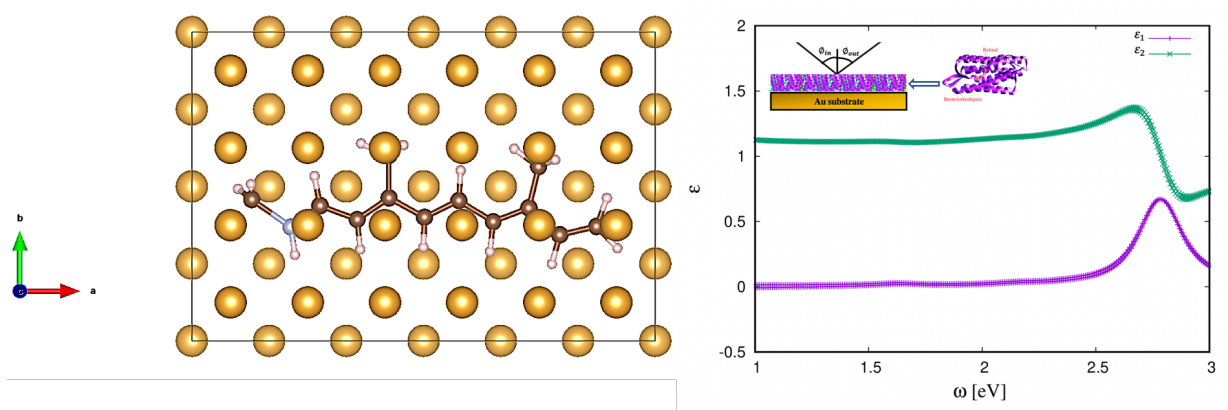

Figure 5: TOCGraphic 